\title{
Aeroacoustic and Aerodynamic Performances of an Aerofoil Subjected to Sinusoidal Leading Edges
}

\author{
Tze Pei Chong ${ }^{1}$, Alexandros Vathylakis ${ }^{2}$, Archie McEwen ${ }^{3}$, Foster Kemsley ${ }^{4}$, Chioma Muhammad ${ }^{5}$ and Saarim \\ Siddiqi ${ }^{6}$
}

Brunel University London, Uxbridge, UB8 3PH, UK

\begin{abstract}
This paper presents the preliminary results on the aeroacoustic and aerodynamic performances of a NACA65-(12)10 aerofoil subjected to 12 sinusoidal leading edges. The serration patterns of these leading edges are formed by cutting into the main body of the aerofoil, instead of extending the leading edges. Any of the leading edges, when attached to the main body of the aerofoil, will always result in the same overall chord length. The experiment was mainly performed in an aeroacoustic wind tunnel facility, although a separate aerodynamic type wind tunnel was also used for the force measurements. These sinusoidal leading edges were investigated for their effectiveness in suppressing the laminar instability tonal noise (trailing edge self-noise) and turbulence-leading edge interaction noise. The largest reduction in aerofoil noise tends to associate with the sinusoidal leading edge of the largest amplitude, and smallest wavelength. However, noticeable noise increase at high frequency is also observed for this combination of serration. In terms of the aerodynamic performance, increasing the serration wavelength tends to improve the stall angles, but the lift coefficient at the pre-stall regime is generally lower than that produced by the baseline leading edge. For a sinusoidal leading edge with large serration amplitude, the effect of the reduction in "lift-generating" surface is manifested in the significant reduction of the lift coefficients and lift curve slope. The sinusoidal leading edge that produces the best performance in the post-stall regime belongs to the largest wavelength and smallest amplitude, where the lift coefficients are shown to be better than the baseline leading edge. In conclusion, large amplitude and small wavelength is beneficial for noise reduction, whilst to maintain the aerodynamic lift a small amplitude and large wavelength is preferred.
\end{abstract}

\section{Introduction}

$\mathrm{W}$ hen the leading edge of an aerofoil is undulated, it may influence the local acoustical response to the incoming flow with freestream turbulence. Flow perturbation by the undulated leading edges, especially at low Reynolds number where the boundary layer is mostly laminar, could also affect the noise scattering at the trailing edge. Leading edge undulations of an aerofoil in the forms of sinusoidal/wavy shape and sawtooth shape can be considered as bio-inspired from the humpback whale tubercles and/or owl wing serration, respectively. Generally, a wavy or sinusoidal leading edge has little distinction between them, but a sawtooth leading edge tends to involve more sharp corners in a zigzag pattern.

Many of the earlier publications on leading edge undulation focused on the aerodynamic performance. Work performed by Fish and Battle [1] has shown that the humpback whale benefits from tubercles (wavy protuberances) along the leading edge of its flippers, enhancing its manoeuvrability by delaying flow separation. The morphology of the humpback whale could benefit the wind turbines and Micro-Air-Vehicles (MAVs). Despite the delayed

\footnotetext{
${ }^{1}$ Lecturer, Department of Mechanical, Aerospace and Civil Engineering, t.p.chong@brunel.ac.uk, AIAA Member

${ }^{3,4,6}$ Undergraduate students, Department of Mechanical, Aerospace and Civil Engineering, Non-AIAA Member

${ }^{2,5} \mathrm{PhD}$ students, Department of Mechanical, Aerospace and Civil Engineering, Non-AIAA Member
} 
aerodynamic stall and improved post-stall lift performance, the wavy leading edges also reduce the maximum lift coefficient and increase the drag coefficient, resulting in low lift-to-drag ratio. Johari et al. [2] performed experimental evaluation of the effects of various wavelength and amplitude configurations on the aerodynamic force characteristics of a full-span NACA $63_{4}-021$ aerofoil at a Reynolds Number of 183,000. Despite the increased poststall lift up to 50\%, wavy leading edges also produce a reduction in both stall angle and lift coefficient, as well as a significant increase in drag coefficient. Miklosovic et al. [3] performed a study of the 2D and 3D flow effects of a wavy leading edge by comparing performance of a full-span model with a half-span model. Improvements in lift coefficient were observed for the semi-span models, however a significant performance reduction was observed for full span models (up to $38 \%$ less lift, $138 \%$ more drag, despite an increase in post-stall lift of up to $48 \%$ ). Evidence of spanwise vortices generated by wavy leading edges from flow visualisation techniques (surface tufts [2] and hydrogen bubble [4]) has been established. These vortices are regarded as the mechanism of boundary layer separation delay and improved post-stall performance. Leading edge undulation in a sawtooth pattern is also studied. The aerodynamic characteristics of a serrated sawtooth strip on the pressure side of an aerofoil were investigated by Soderman et al. [5] where reduction of separation bubble size was observed. Small increases in the maximum lift coefficient and reduction in drag coefficient were also found.

Recently there is renewed interest to apply leading edge undulation to achieve aerofoil turbulence-leading edge interaction noise reduction. The effect of the leading edge device originally tested by Soderman et al. [5] was further investigated by Hersh and Hayden [6] for its ability to reduce aerofoil noise from helicopter blades. They showed that leading edge serrations can achieve noise reduction between 4-8 dB. In a joint experimental-numerical investigation by Clair et al. [7] on the leading edge interaction noise of a NACA 65-(12)10 aerofoil, the wavy leading edge is found to achieve noise reduction between 3-4 dB. Narayanan et al. [8] assess the effects of a serrated leading edge on the turbulence-leading edge interaction noise produced by a flat plate and the same NACA65 series aerofoil. A near isotropic turbulence was produced using mesh grid inside the nozzle. They demonstrated significant noise reduction of $9 \mathrm{~dB}$ by flat plate and $7 \mathrm{~dB}$ by aerofoil configurations, respectively. They suggest that the level of broadband noise reduction is a strong function of the amplitude (distance between the peak and trough), and is less sensitive to the serration wavelength (distance between successive peaks).

Apart from the turbulence-leading edge interaction noise, leading edge undulation can also influence the noise spectrum radiated from the trailing edge. In fact, the observed noise reduction in the earlier work of Soderman et al. [5] is likely to be associated with the laminar tonal self-noise scattered from the trailing edge. Recently, Hansen et al. [9] adopted sinusoidal leading edges on a NACA 0021 aerofoil. They show some modifications of the laminar instability tone in the sound pressure level and the tone frequency. Similarly, the level of tonal noise reduction was dependent on both the amplitude and wavelength of the serration. The underlying principle for the modification of the tonal noise radiated from the trailing edge is likely to be caused by the interaction between the spanwise array of vortices (shed from the troughs) and the boundary layer separation bubble. It was also suggested that the undulated leading edge confines separation to the troughs, thereby reducing the susceptibility of a boundary layer to external acoustic excitation.

Undulating the frontal part of an aerofoil has been shown to be able to influence the aerodynamic and aeroacoustic performances of an aerofoil. In order to connect the two key performance metrics, this paper will present both the aerodynamic and aeroacoustic results of a NACA 65-(12)10 aerofoil subjected to sinusoidal leading edges. This type of aerofoil was also studied previously in [7,8]. A total 12 sinusoidal leading edges, plus one straight leading edge to serve as the baseline case, were investigated in this study. The experimental setup, including brief descriptions of the experimental techniques, will be presented in Section II. For the noise results, the first to present is related to the bare jet case where no turbulence grid is used. Under this configuration the main noise source for a baseline aerofoil is the trailing edge self-noise where boundary layer instabilities scattered into tonal noise. The second case is related to the turbulence-leading edge interaction noise where a turbulence grid is used to generate a near isotropic turbulence in the freestream. All the noise results will be presented in Section III. Lift coefficients and flow visualisation on the aerofoil's suction side subjected to some of the sinusoidal leading edges will be discussed in Section IV. The paper then concludes in Section V.

\section{Experimental setup}

\section{A. Wind tunnel facilities and instrumentations}

Free field measurements of the aerofoil noise were mainly conducted in the aeroacoustic wind tunnel at Brunel University London, which is situated in a $4 \mathrm{~m} \times 5 \mathrm{~m} \times 3.4 \mathrm{~m}$ anechoic chamber. As shown in Figs. 1a and 1b, the nozzle exit is rectangular with dimensions of $0.10 \mathrm{~m}$ (height) $\mathrm{x} 0.30 \mathrm{~m}$ (width). This wind tunnel can achieve a 
turbulence intensity of between $0.1-0.2 \%$. The background noise of the wind tunnel facility is well below the selfnoise of the quietest aerofoil across the whole range of velocity [10]. The range of jet speeds under investigation was between $20 \mathrm{~ms}^{-1}$ and $60 \mathrm{~ms}^{-1}$, corresponding to Reynolds numbers based on aerofoil chord, $C$ of $2 \times 10^{5}$ and $6 \times 10^{5}$ respectively. The aerofoil was held by side plates and attached flushed to the nozzle lips. Unless otherwise stated, the aerofoil was positioned at zero degree angle of attack during the aeroacoustic experiments. As shown in Fig. 1b, far field noise measurements were made by a single condenser microphone at a distance of $1.0 \mathrm{~m}$ from the aerofoil mid-chord and mid-span. The reason the microphone was placed at the mid-chord is due to the consideration that trailing edge noise and leading edge noise will be produced by the bare jet and turbulence grid cases, respectively. The position of the microphone thus produces polar angles of $94^{\circ}$ and $86^{\circ}$ with relative to the trailing edge and leading edge, respectively. Noise data from the microphone was acquired at a sampling frequency of $44 \mathrm{kHz}$ for 10 seconds by a 16-bit Analogue-Digital card from National Instrument. The data was then windowed and the Power Spectral Density (PSD) of $1 \mathrm{~Hz}$ bandwidth computed from a 1024 point FFT.

Aerodynamic force measurements were made in a conventional wind tunnel with closed-working section. This wind tunnel is not acoustically treated and is not suitable for noise measurement. Instead, it is only used for measurements of the lift and drag produced by the aerofoil with sinusoidal trailing edges. Note that the drag results will not be discussed here for brevity, but they will be presented in our subsequent publications. The wind tunnel has a test section of $0.5 \mathrm{~m} \times 0.5 \mathrm{~m}$, a maximum velocity in the test section of about $38 \mathrm{~ms}^{-1}$ and a freestream turbulence intensity of about $0.2-0.3 \%$. The aerofoil model was mounted horizontally across almost the entire width of the test section (1 mm gap on each side of the wind tunnel side window). In order to investigate the effect of the sinusoidal leading edges on the aerodynamic forces, a 3-component strain gauge force balance was used to measure the aerodynamic forces produced by the aerofoil. As shown in Fig. 1c, it consists of a mounting plate, which is used to secure the device to the wind tunnel side window, and a triangular force plate. The force plate and the mounting plate were connected via three spherical universal joints constraining the motion of the force plate parallel to the direction of the mounting plate. Forces incurred by the aerofoil were transmitted to three strain gauges via the cables. The angle of attack of the airfoil was rotated via a disc between $-8^{\circ}$ and $+30^{\circ}$. Wind tunnel blockage correction was applied in the data analysis.

Oil flow visualisation was conducted on the suction side of the aerofoil surface to examine the dynamic changes of near wall properties subjected to the sinusoidal leading edges. The oil mixture used in this study consists of three components: linseed oil providing the oil base of the compound, titanium dioxide as a colouring agent, and paraffin for controlling the viscosity of the compound. Care was taken to obtain an appropriate viscosity of the compound. It should be noted that application of the oil compound to the aerofoil surface is unlikely to affect the boundary layer significantly, especially when observing the large scale vortex formation from the sinusoidal leading edges.

\section{B. Sinusoidal leading edges and aerofoil model}

As shown in Fig. 2, the aerofoil under investigation here is a NACA 65-(12)10, cambered aerofoil. The chord length, $C$ is $150 \mathrm{~mm}$, and the width is $498 \mathrm{~mm}$. The sinusoidal leading edges were cut directly into the main body of the aerofoil, thus preserving the aerofoil chord length. Between the leading edge $x / C=0$, and $x / C=0.33$, is a section that can be removed and replaced by different sinusoidal profiles. Note that $x$ is the streamwise direction. Further downstream, $0.33<x / C \leq 1.0$, is the unmodified aerofoil main body. Once attached the sinusoidal leading edge a continuous profile is formed giving the appearance that the sinusoidal patterns are cut into the main body of the aerofoil. A total 12 sinusoidal leading edge sections, plus one with straight leading edge to serve as a baseline case, were investigated in this study.

When describing the sinusoidal pattern, two parameters are normally used as per the Fig. 2: the wavelength, $\lambda$ and the amplitude, $a$. The values of $\lambda$ investigated in this study are $7.5,15,30$ and $45 \mathrm{~mm}$, which give $\lambda / C=0.05$, $0.1,0.2$ and 0.3 , respectively. A similar range also applies to the amplitude where $a / C=0.05,0.2$ and 0.3 . A summary of the sinusoidal leading edges can be found in Fig. 2.

\section{Grid generated turbulence}

A woven wire mesh screen is well known for its ability to reduce turbulence intensity and improve flow steadiness. Under normal circumstances, this type of device consists of small mesh length $M$ and wire diameter $d$. These parameters are depicted in Fig. 3. Some combinations of $M$ and $d$ in a bi-planar grid, as well as the flow velocity, however, can produce exactly the opposite effect: increasing the freestream turbulence intensity downstream of the device. A large value of $d$ tends to encourage stronger vortex shedding, thereby increasing the level of turbulence intensity. However, different values of $M$ and velocity with the same $d$ can also produce very different turbulence intensity level, and possibly the integral length scale of the turbulent eddies. Recommendation by Laws and Livesey [11] of a mesh-to-diameter ratio, $M / d=5$ for turbulence generated by a bi-planar orthogonal 
square grid represents a good reference. This criterion is adopted in the current study where $M=75 \mathrm{~mm}$ and $d=15$ $\mathrm{mm}$. The grid is placed inside the nozzle. The accelerating flow inside the nozzle could potentially re-laminarise the grid-generated turbulence. To minimise this effect the turbulence grid was placed after the largest curvature point as shown in Fig. 3.

The decay of the turbulence intensity level in the streamwise distance downstream of the grid, $x_{l}$ is the function of $\left(x_{l}-x_{o}\right)^{-5}$, where $x_{o}$ is the virtual origin of the grid. As a rule of thumb, about twenty times the mesh length $M$ is required to achieve isotropic turbulence behind the grid. This criterion is difficult to be fulfilled in the current study due to the restrictive geometry between the nozzle exit and the aerofoil model. Hot-wire anemometry was used to examine the isotropy of the grid-generated turbulence. Flow measurement was conducted by a hot wire probe at location near the aerofoil leading edge (without the presence of the aerofoil during the measurement). The measured velocity power spectral density was then compared with the Liepmann model. The dilution in the high frequency region caused by the Kolmogorov scale is also compensated by applying an exponential function [12]. As shown in Fig. 3, at flow velocity $30 \leq U \leq 60 \mathrm{~ms}^{-1}$, the measured streamwise velocity spectra demonstrate $-5 / 3$ decay rate and with acceptable agreement to the Liepmann model, except at the low frequency region. The measured streamwise velocity turbulence intensity at location near the aerofoil leading edge is $3.7 \%$, and the integral length scale is about $6.5 \mathrm{~mm}$.

\section{Noise Results}

\section{A. No grid case: low turbulence jet}

The first aeroacoustic results to be discussed are related to the case when no grid is installed inside the nozzle. The radiated noise from a baseline aerofoil (i.e. with a straight leading edge) has its origin at the trailing edge, as demonstrated by the Sound Pressure Level, SPL contour map in Fig. 4a. Several familiar features pertaining to the laminar instability tonal noise are discernible: 1) the existence of velocity scaling laws $\sim U^{1.5}$ and $U^{0.8}$ for the broadband hump and discrete tones, respectively $[13,14]$; and 2) the existence of the "ladder" structure. The result indicates that bypass boundary layer transition on the aerofoil surfaces was not triggered due to the low freestream turbulence intensity. To confirm this, a boundary layer trip tape was placed at $x / C=0.88$ on the aerofoil's suction side. The reason to place the trip tape at a relatively downstream location is to ensure that the triggered turbulent boundary layer, if any, will not produce significant broadband noise level. It was shown in Fig. 4b that the trip tape completely suppresses the laminar instability tonal noise, which could be due to the absence of laminar separation bubble in this case. The results in Fig. 4 also demonstrate that leading edge interaction noise is not significant.

Next, the effectiveness of the sinusoidal leading edge in suppressing the laminar instability tonal noise is investigated. In all these cases no trip tape was used on the aerofoil surface. Figure 5a compares the SPL spectra of four sinusoidal leading edges (same $a / C$ at 0.05 , but different $\lambda / C$ at $0.3,0.2,0.1$ and 0.05 ) with the baseline aerofoil. Improved noise reduction is demonstrated when $\lambda / C$ is decreased. The frequencies of the multiple discrete tones remain the same, which indicate that flow structures generated by the sinusoidal leading edges (i.e. streamwise vortices as shown in Section IV.B) may not alter the boundary layer instabilities significantly. The likely explanation is that more streamwise vortices are generated per unit span when $\lambda / C$ is small, thus they are more effective in suppressing the laminar separation bubble and destroying the amplification process with the incoming boundary layer instabilities. This is supported by comparing the surface oil flow results in Figs. $5 \mathrm{c}, 5 \mathrm{~d}$ and $5 \mathrm{e}$, which were obtained under the same flow speed using the same aeroacoustic wind tunnel. For the baseline straight leading edge in Fig. 5c, separation region is visible near the trailing edge as indicated by the two dotted lines. For the sinusoidal leading edge of $\lambda / C=0.3$ and $a / C=0.05$ in Fig. $5 \mathrm{~d}$, streamwise vortices were generated at the troughs but they largely fail to penetrate the separation region. As a result the oil residues were accumulated upstream of the separation line forming several ring shapes. This corresponds well with the noise result in Fig. 5a where a significant tonal noise spectrum is still present in this case. When the sinusoidal leading edge is replaced with $\lambda / C=0.1$ and $a / C$ $=0.05$ in Fig. $5 \mathrm{e}$, more streamwise vortices were generated per unit span but they are still not able to influence the separation region significantly. As a result, tonal feature is still present in Fig. 5a, although the magnitude is now lower in this case.

Generally, a sinusoidal leading edge with a small $a / C$ is not desirable. Figure $5 \mathrm{~b}$ compares the SPL spectra of three sinusoidal leading edges (same $\lambda / C$ at 0.30 , but different $a / C$ at $0.05,0.2$ and 0.3 ) with the baseline aerofoil. The results show that significant tonal noise reduction can be achieved with a large $a / C$. As shown in Fig. $5 \mathrm{f}$ for the $\lambda / C=0.3$ and $a / C=0.3$ sinusoidal leading edge, strong streamwise vortices were generated at the troughs that propagate up to the trailing edge, thus suppressing the separation region locally. Because of the relatively large 
wavelength, and that vortices are not generated at the peak regions, pockets of flow separation are still present near the trailing edge. However, when the sinusoidal leading edge is replaced with the $\lambda / C=0.1$ and $a / C=0.3$ type where more streamwise vortices are generated per unit span, the separation region near the trailing edge is almost completely suppressed as shown in Fig. $5 \mathrm{~g}$. As a result, the reduction in tonal noise is even greater as manifested in Fig. 5b.

\section{B. Grid generated turbulence}

This section concerns the case when the turbulence grid described in Section II.C is installed inside the nozzle. Figure 6 compares the SPL spectra for the 12 sinusoidal leading edges with the baseline aerofoil at $U=24$ and 58 $\mathrm{ms}^{-1}$. Note that trip tape was not used on the aerofoil surface. By examining the SPL spectra produced by the baseline aerofoil, it is clear that they are mostly broadband in nature, which indicates that the main noise source is now the turbulence-leading edge interaction noise. It is also postulated that the elevated freestream turbulence intensity triggered a bypass transition on the aerofoil surface, as there is no trace of instability tonal noise in the spectra.

Introducing various degrees of $\lambda / C$ and $a / C$ to the sinusoidal leading edges could produce very different noise performances. Whilst the noise performance improves slightly with reducing $\lambda / C, a / C$ is shown to be the main parameter to achieve significant reduction in the leading edge interaction noise. Up to $12 \mathrm{~dB}$ noise reduction is demonstrated for the particular leading edge of $\lambda / C=0.05 \& a / C=0.3$ (smallest wavelength $\&$ largest amplitude). On the other scale of the geometrical parameters of $\lambda / C=0.3 \& a / C=0.05$ (largest wavelength $\&$ smallest amplitude), the corresponding SPL spectra are almost identical to that produced by the baseline aerofoil with straight leading edge.

A better presentation of the interaction noise reduction could be shown by the $\Delta$ SPL contour map in Fig. 7, where $\triangle$ SPL is the difference in SPL produced by the aerofoil with a straight leading edge and a sinusoidal leading edge. Positive values of $\Delta$ SPL denote noise reduction, and vice versa. Results in Fig. 7 largely follow the trend of the SPL in Fig. 6. For the optimal sinusoidal leading edges (smallest wavelength \& largest amplitude), high level of broadband noise reduction over a large frequency bandwidth can be achieved. Note that noise increase at frequency greater than $10 \mathrm{kHz}$ can be observed in some cases.

The $\Delta$ SPL contour maps also exhibit two types of velocity dependence for the noise reduction. One is associated with low-to-medium (and possibly up to the highest velocity of $U=60 \mathrm{~ms}^{-1}$ ) velocity region where large noise reduction follows $\sim U^{1.1}$, while another is only exclusive associated with high velocity region where noise reduction follows $\sim U^{0.4}$. These velocity dependencies exist for almost all the sinusoidal leading edges investigated here.

The Overall Sound Pressure Level, OASPL is another useful quantity to appraise the noise reduction performances of the sinusoidal leading edges. Bar charts of $\triangle$ OASPL (difference in OASPL produced by the aerofoil with a straight leading edge and a sinusoidal leading edge) are shown in Figs. 8a and $8 \mathrm{~b}$ at $U=24$ and 58 $\mathrm{ms}^{-1}$, respectively. Note that when integrating the mean square acoustic pressure to obtain the OASPL, the lower and upper limits of the frequencies are 50 and $20,000 \mathrm{~Hz}$, respectively. Again, the $\triangle \mathrm{OASPL}$ bar charts also demonstrate the superior noise reduction performance with a small-wavelength and large-amplitude sinusoidal leading edge. When comparing the two smallest wavelengths of $\lambda / C=0.1$ and 0.05 in the $\triangle \mathrm{OASPL}$, the results also suggest that the $\lambda / C=0.05$ case is not necessary the most optimum choice. This might be due to the tendency of high frequency noise increase for the small wavelengths (see Fig. 7).

The results presented thus far are associated with the aerofoil when it is fixed at zero degree angle of attack. Next we will examine the noise performance when the aerofoil is set at different angles of attack between $-8^{\circ}$ and $+10^{\circ}$. Note that these are the geometrical angles of attack in the context of testing in open jet wind tunnel. The effective angles of attack will be different due to the jet deflection. Nevertheless, it is not essential in the present work to know the exact values of the effective angles of attack. Figure 9 shows the slices of contour maps at different $U$, frequency and geometrical angles of attack. It can be seen that noise reduction becomes less effective at negative angle of attack, and slightly better at positive angle of attack. The best reduction actually occurs at zero degree angle of attack. To explain this, three schematics were drawn alongside. At negative angle of attack, the stagnation point is on the suction surface where the main flow is deflected upwards, which then misses the valleys of the serration. The same can be applied to the large positive angle of attack case where the stagnation point is at the pressure surface, though there are still certain extents of main flow passing through the serration valley. At zero angle of attack, the stagnation point is close to the leading edge noise. In this case, the main flow impinges the leading edge where the serration can exert its "turbulence filtering" effect to reduce the interaction noise. The range of geometrical angle of attack $\left(-8^{\circ}\right.$ and $\left.+10^{\circ}\right)$ investigated here is likely to yield a smaller range of effective angle of attack. This implies that the noise performance of the sinusoidal leading edge will be sensitive to the angle of attack. 


\section{Aerodynamic Results}

\section{A. Lift measurement}

This section shows the lift coefficients, $C_{L}$ for several types of sinusoidal leading edges at different angles of attack. The aerodynamic results were obtained at a separate wind tunnel described earlier. The freestream velocity was set at $15 \mathrm{~ms}^{-1}$, corresponding to the Reynolds number of 150,000 based on $C$. Note that the same planform area was used throughout the analysis of the $C_{L}$, regardless it is a baseline leading edge or a sinusoidal leading edge.

Figure 10a compares the $C_{L}$ produced by three sinusoidal leading edges $(a / C=0.3,0.2$ and 0.05 , all with $\lambda / C=$ $0.10)$ and the baseline leading edge aerofoil. $C_{L}$ predicted by the XFOIL package, which is obviously only related to a straight leading edge, is also included. Both the experimental and numerical results match pretty well, and both exhibit a lift curve slope (at the pre-stall region) of about 0.1 per degree. The $C_{L}$ produced by the sinusoidal leading edge of $a / C=0.3$ and $\lambda / C=0.10$ are significantly lower than those produced by the baseline aerofoil. The reason of a reduced $C_{L}$ is likely to be caused by the considerable loss of lift-generating surfaces in this case. The corresponding lift curve slope drops to 0.062 per degree. The stall angle is slightly better, and the drop in $C_{L}$ with angle of attack at the post-stall region is more gradual than the baseline leading edge. The lift performance improves slightly with $a / C=0.20$ and $\lambda / C=0.10$, where the serration amplitude is smaller. Interestingly, at the smallest serration amplitude of $a / C=0.05$ and $\lambda / C=0.10$, the recovery in aerodynamic performance is quite remarkable. For such a small serration amplitude, the lift curve slope recovers to 0.093 per degree, the stall angle is considerably larger and the drop in $C_{L}$ after the stall angle is more gentle than the baseline leading edge. As a result, this type of sinusoidal leading edge has a superior performance at the post-stall regime.

Figure 10b compares the $C_{L}$ of other three sinusoidal leading edges with larger wavelength $(a / C=0.3,0.2$ and 0.05 , all with $\lambda / C=0.20$ ). The results in Fig. $10 \mathrm{~b}$ demonstrate a better performance in lift and the lift curve slope compared to those Fig. 10a, indicating that a larger wavelength is another criterion to maintain the aerodynamic performance. Remarkably, the one with the smallest serration amplitude $a / C=0.05$ and $\lambda / C=0.20$ even produces a larger $C_{L}$ than the baseline leading edge at above 12 degree angle of attack. The $C_{L \max }$ (maximum lift coefficient) for the sinusoidal leading edge is also compatible with the baseline leading edge.

Summary of the lift curve slopes for the baseline and sinusoidal leading edges can be found in Table 1 .

\section{B. Surface flow visualisation}

This section shows a selective of the surface oil flow visualisation results. As shown in Fig. 11, surface flow topologies produced by the baseline leading edge is shown alongside with two sinusoidal leading edges $(\lambda / C=0.2 \&$ $a / C=0.05)$ and $(\lambda / C=0.2 \& a / C=0.3)$ at 10 degree angle of attack. For the baseline leading edge in Fig. 11a, boundary layer separation is apparent near at the aft region of the suction side of the aerofoil (indicated by the dash red line). When the leading edge is replaced with the $(\lambda / C=0.2 \& a / C=0.05)$ in Fig. 11b, a pair of streamwise vortices seem to emanate on each trough. Due to the counter-rotating of the vortices, mutual inductions between adjacent vortices are demonstrated in the magnified picture associated with Fig. 11b. Owing to the small serration amplitude $a / C=0.05$ in this case, the vortices seem to be only able to weakly energise the separated boundary layer. As a result, separation bubble is still visible near the trailing edge across the span.

When the leading edge is replaced with the $(\lambda / C=0.2 \& a / C=0.3)$ in Fig. $11 \mathrm{c}$, the strong streamwise vortices produced by the large serration amplitude seem to be able to penetrate deep into the separated boundary layer up to the trailing edge across the spanwise direction, leaving only a small pocket of separated flow as indicated. A closer look at the vortex shedding generated in this case also reveals that only a single and stronger streamwise vortex is generated at each trough. In this particular, boundary layer separation on the suction side of the aerofoil is almost completely suppressed.

\section{Conclusions}

This paper presents the preliminary results on the aeroacoustic and aerodynamic performances of a NACA65(12) 10 aerofoil subjected to 12 sinusoidal leading edges. The serration patterns of these leading edges are formed by cutting into the main body of the aerofoil, instead of extending the leading edges. Any of the leading edges, when attached to the main body of the aerofoil, will always result in the same overall chord length. The experiment was mainly performed in an aeroacoustic wind tunnel facility, although a separate aerodynamic-type wind tunnel was also used for the force measurements. These sinusoidal leading edges were investigated for their effectiveness in 
suppressing the laminar instability tonal noise (trailing edge self-noise) and turbulence-leading edge interaction noise. The largest reduction in aerofoil noise tends to associate with the sinusoidal leading edge of the largest amplitude, and smallest wavelength. However, noticeable noise increase at high frequency is also observed for this combination of serration. In terms of the aerodynamic performance, increasing the serration wavelength tends to improve the stall angles, but the lift coefficient at the pre-stall regime is generally lower than that produced by the baseline leading edge. For a sinusoidal leading edge with large serration amplitude, the effect of the reduction in "lift-generating" surface is manifested in the significant reduction of the lift coefficients and lift curve slope. The sinusoidal leading edge that produces the best performance in the post-stall regime belongs to the largest wavelength and smallest amplitude, where the lift coefficients are shown to be better than the baseline leading edge. In conclusion, large amplitude and small wavelength is beneficial for noise reduction, whilst to maintain the aerodynamic lift a small amplitude and large wavelength is preferred.

\section{Acknowledgments}

The authors would like to thank Mr Till Biedermann and Mr Jay Gadhvi for their helps in some of the measurements.

\section{References}

[1] Fish.F and Battle.J. Hydrodynamic design of the Humpback Whale Flipper. Journal of Morphology, 225 (1995) 51-60.

[2] Johari.H, Henoch.C, Custodio.D, and Levishin.A. Effect of Leading Edge Protuberances on Airfoil Performance. AIAA Journal, 45 (2007) 2634-2642.

[3] Miklosovic.D.S, Murray.M.M, Howle.L.E, and Fish.F.E. Leading Edge Tubercles delay stall on a Humpback Whale Flipper. Physics of Fluids, 16 (2004) L39-L42.

[4] Hansen.K.L, Kelso.R.M, and Dally.B.B. Performance variations of Leading Edge Tubercles for Distinct Airfoil Profiles. AIAA Journal, 49 (2011) 185-194.

[5] Soderman.P. Aerodynamic effects of Leading Edge Serrations on a two dimensional Airfoil. NASA Technical Memorandum (1972) TM X2643.

[6] Hersh.A.S and Hayden.R.E. Aerodynamic sound radiation from lifting surfaces with and without leading-edge serrations. NASA Contract Report (1971) No. 114370.

[7] Clair.V, Polacsek.C, Le Garrec.T, Reboul.G, Gruber.M and Joseph.P.F. Experimental and numerical investigation of turvulence-airfoil noise reduction using wavy edges. AIAA Journal, 51 (2013) 2695-2713.

[8] Narayanan.S, Joseph.P.F, Haeri.S, Kim.J.W, Chaitanya.P, and Polacsek.C. Noise reduction studies from the leading edge of serrated flat plates. 20 $0^{\text {th }}$ AIAA/CEAS Aeroacoustic Conference, AIAA-2014-2320, Atlanta, USA

[9] Hansen.K, Kelso.R, and Doolan.C. Reduction of flow induced airfoil tonal noise using leading edge sinusoidal modifications. Acoustics Australia, 40 (2012) 172-177.

[10] Vathylakis.A, Kim.J.H and Chong.T.P. Design of a low-noise aeroacosutic wind tunnel facility at Brunel University. 20 ${ }^{\text {th }}$ AIAA/CEAS Aeroacoustic Conference, AIAA-2014-3288, Atlanta, USA.

[11] Laws, E. M., Livesey, J. L. Flow Through Screens, Annu. Rev. Fluid Mech. 10 (1978) 247-266

[12] Rozenberg, Y.: Modélisation analytique du bruit aérodynamique à large bande des machines tournantes : utilisation de calculs moyennés de méanique des fluids, Acoustics. Ecole Centrale de Lyon, Lyon, France: 2007.

[13] Paterson, R., Vogt, P., Fink, M. and Munch, C., "Vortex Noise of Isolated Airfoils," Journal of Aircraft, Vol. 10, 1973, pp. $296-302$.

[14] Chong.T.P and Joseph.P.F. An experimental study of airfoil instability tonal noise with trailing edge serrations. Journal of Sound and Vibration, 332 (2013) 6335-6358. 


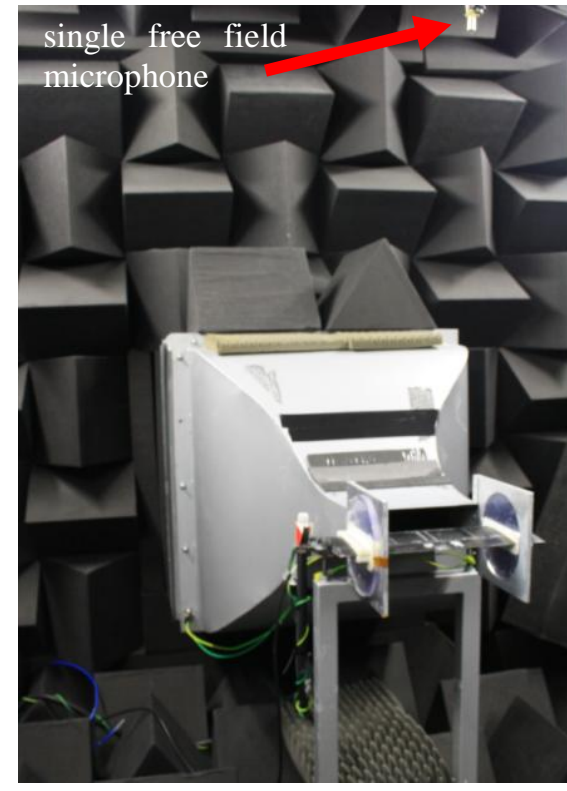

a)

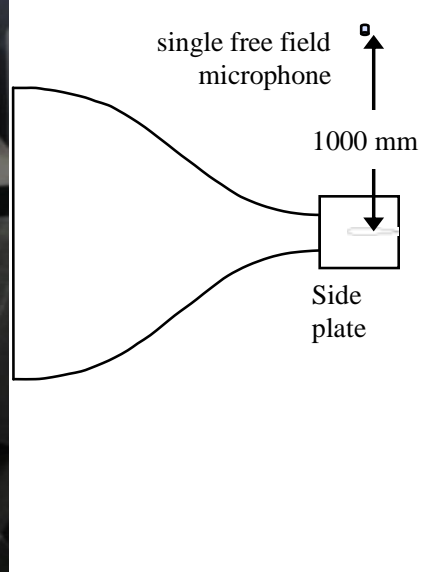

b)

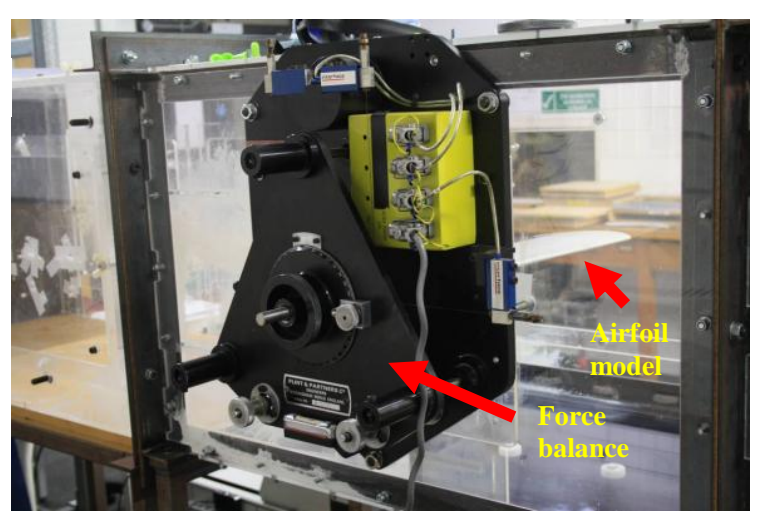

c)

Fig. 1 a) Photograph showing the experimental setup for the aeroacoustic measurements, b) microphone location with relative to the aerofoil, and c) force balance used for lift and drag measurements of the aerofoil in an aerodynamic wind tunnel. 


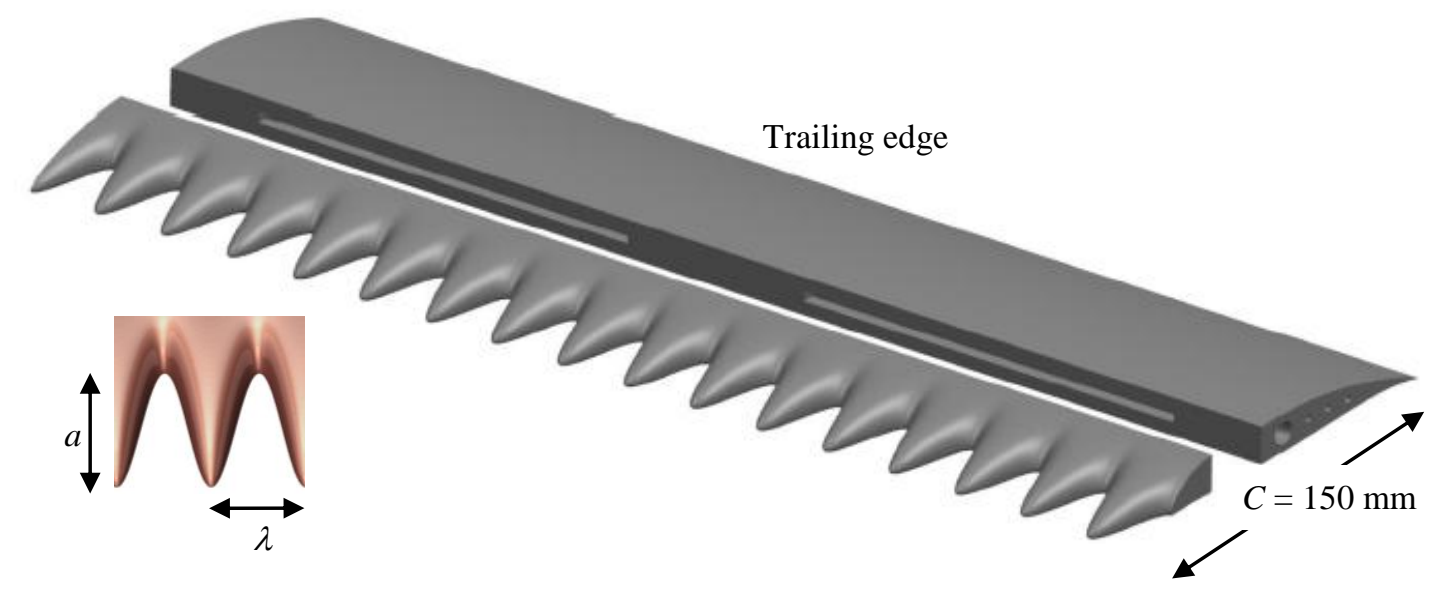

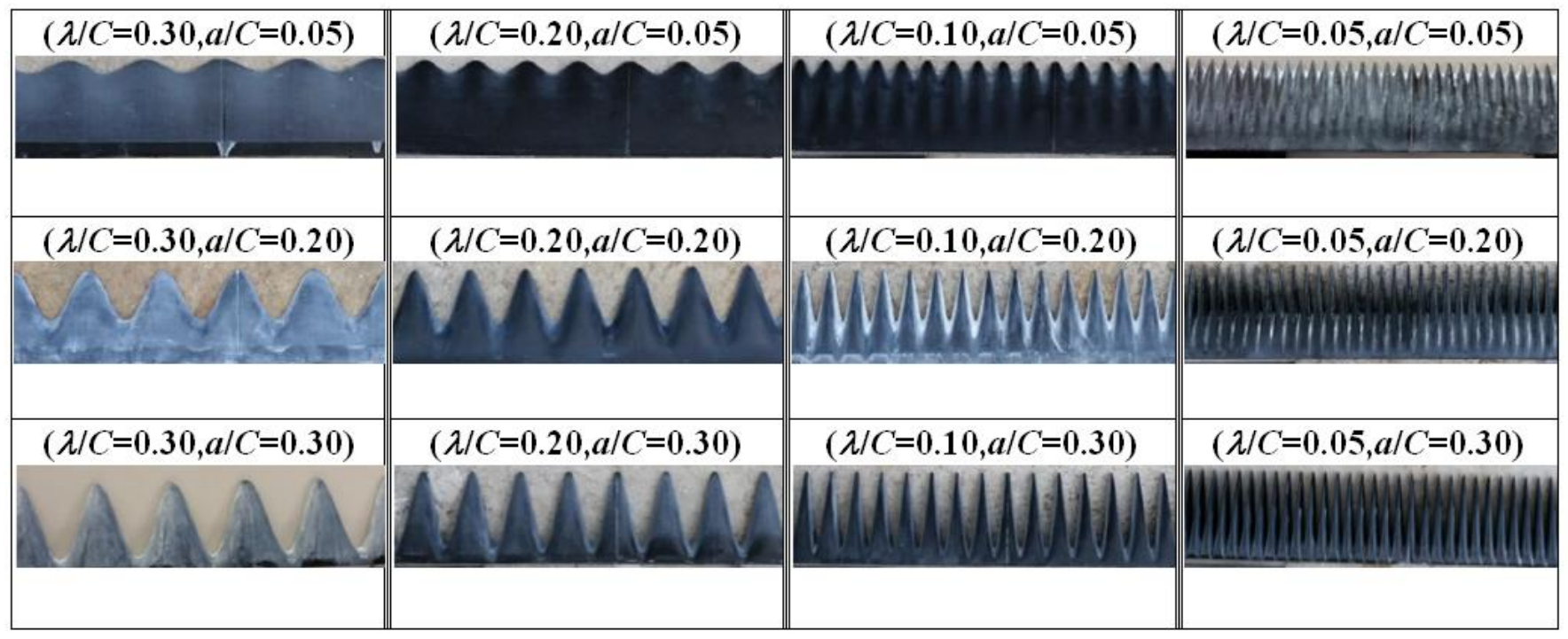

Fig. 2 Illustrations of the NACA65(12)-10 aerofoil and the sinusoidal leading edges 

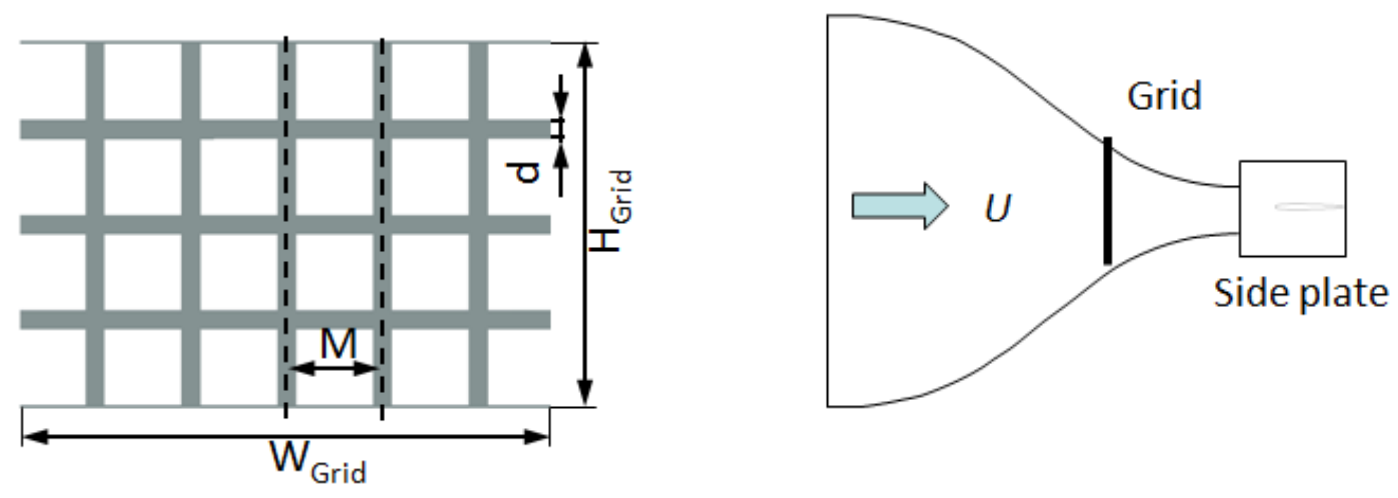

$$
\Phi_{\text {uu }}(\omega)=\frac{\overline{u^{2}} \Lambda_{u u}}{\pi U} \times \frac{1}{1+K_{x}^{2} \Lambda_{w u}^{2}} \cdot G, \quad K_{x}=\frac{\omega}{U}
$$

$$
G=\exp \left((-9 / 4) \cdot\left(K_{x} / K_{\eta}\right)^{2}\right), \quad K_{\eta} \approx 4.8 \times 10^{4} m^{-1}
$$

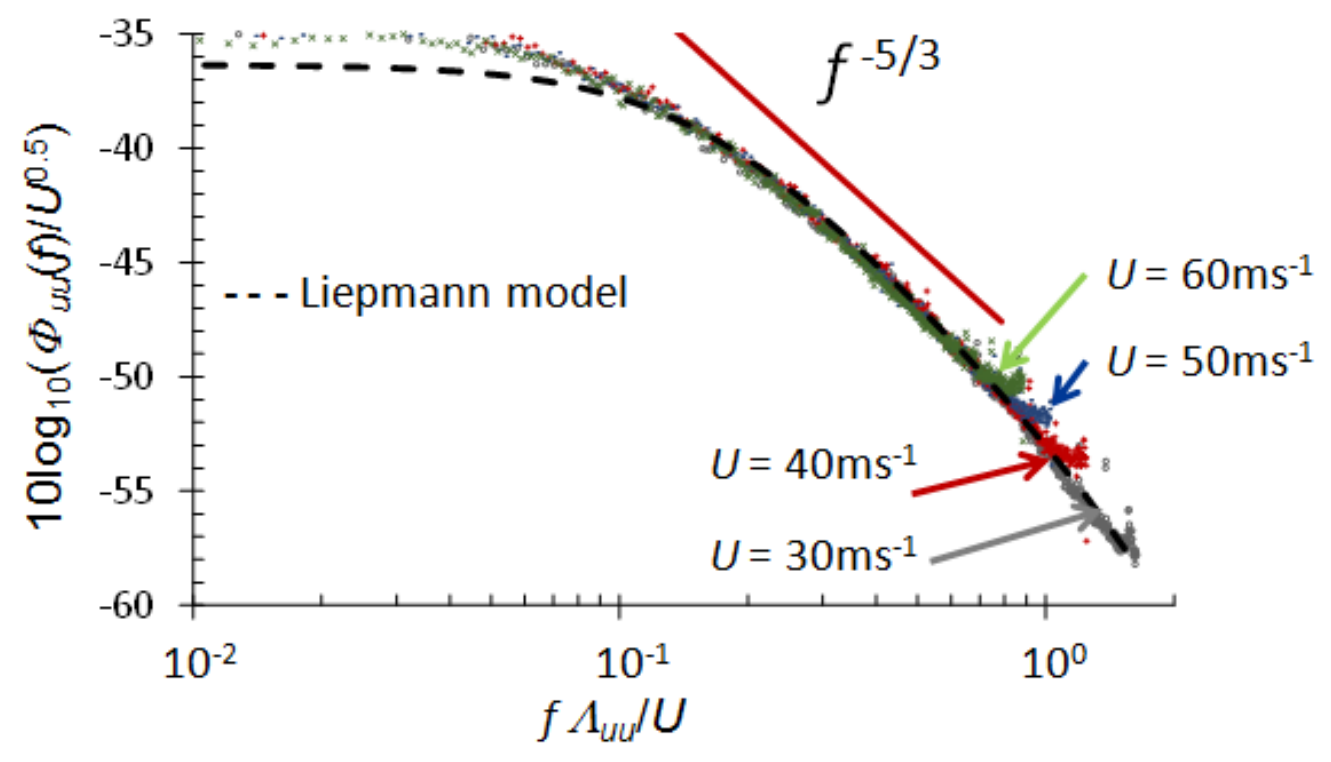

Fig. 3 Comparison of velocity power spectral density with the Liepmann model. $U=30$ $60 \mathrm{~ms}^{-1}$ in steps of $10 \mathrm{~ms}^{-1}$. The integral length scale $\Lambda_{u u}$ (obtained from measured velocity at each speed) was used as the scaling parameter. 


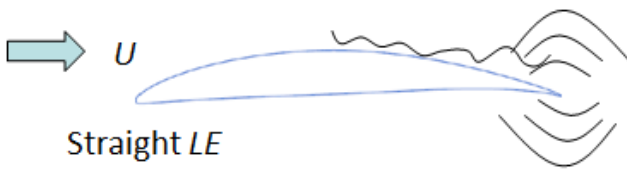

a)

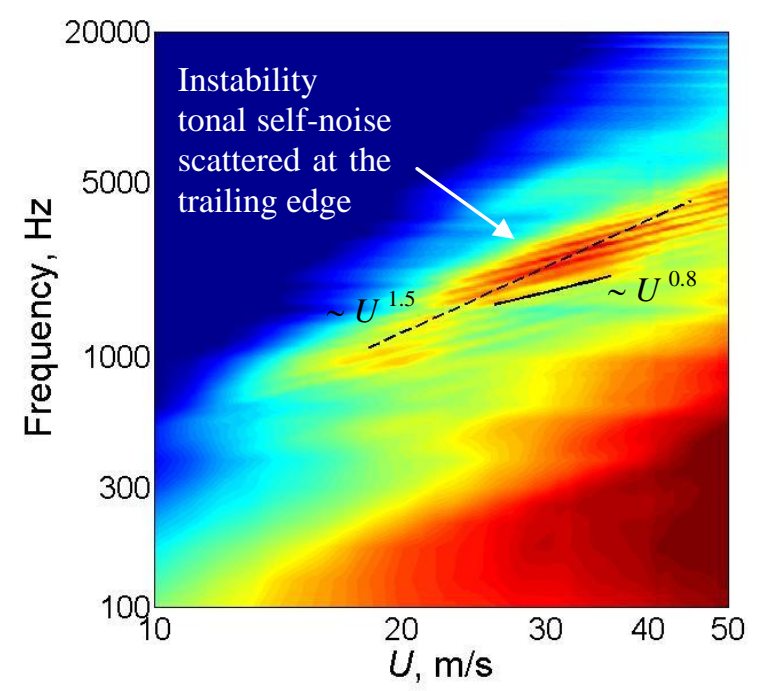

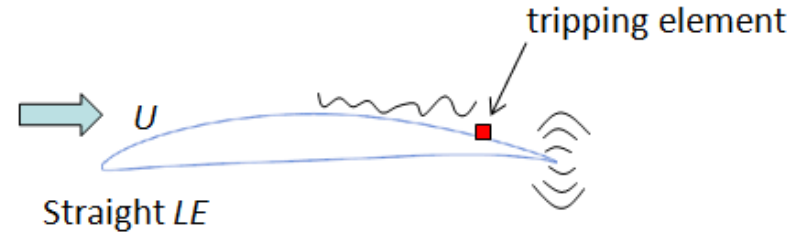

b)

SPL, dB

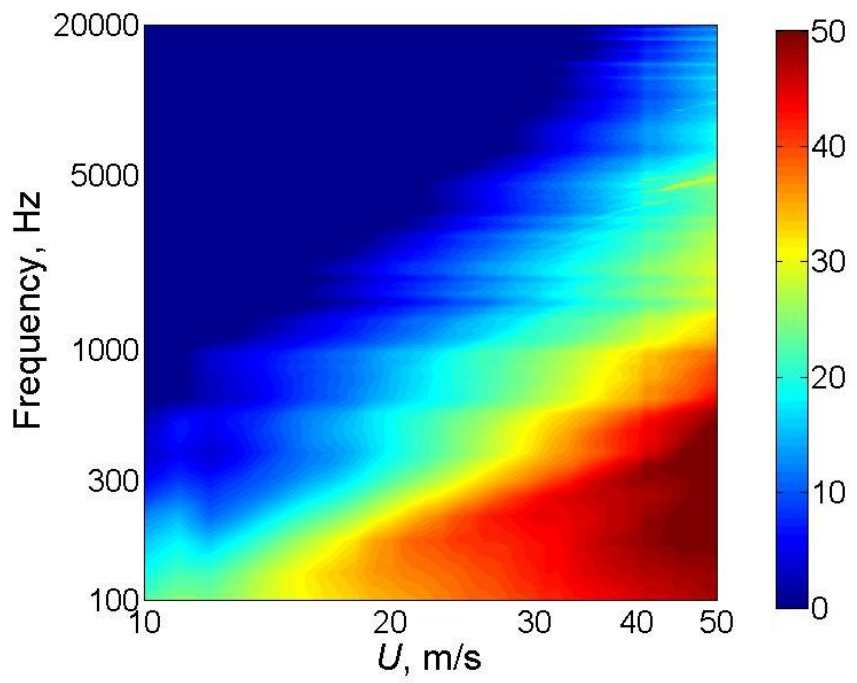

Fig. 4 Contour maps of SPL, dB at different velocities and frequencies, no turbulence grid, produced by the baseline straight leading edge when the boundary layer at the aerofoil suction side was a) untripped and b) tripped at $x / C=0.88$. 
a)

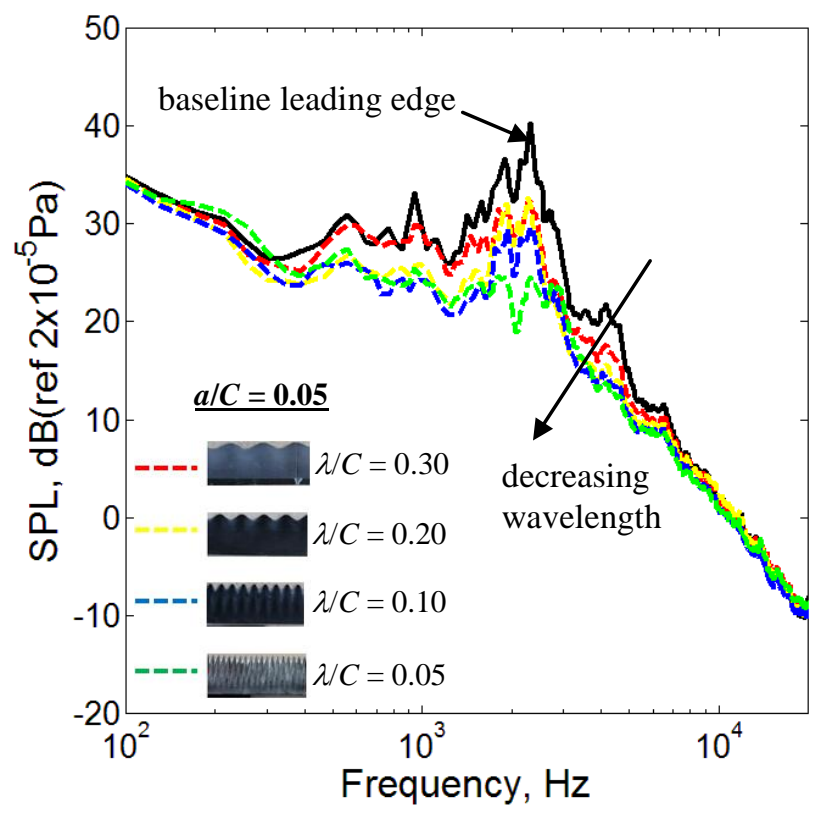

d)

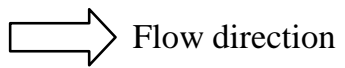

c)

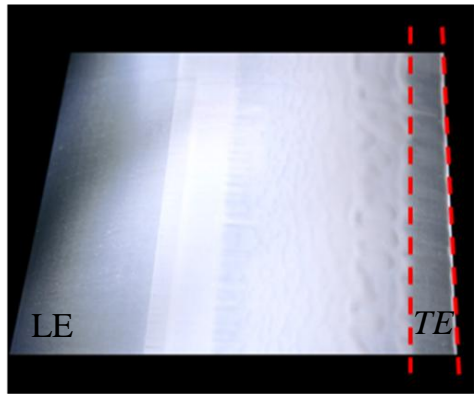

f) b)

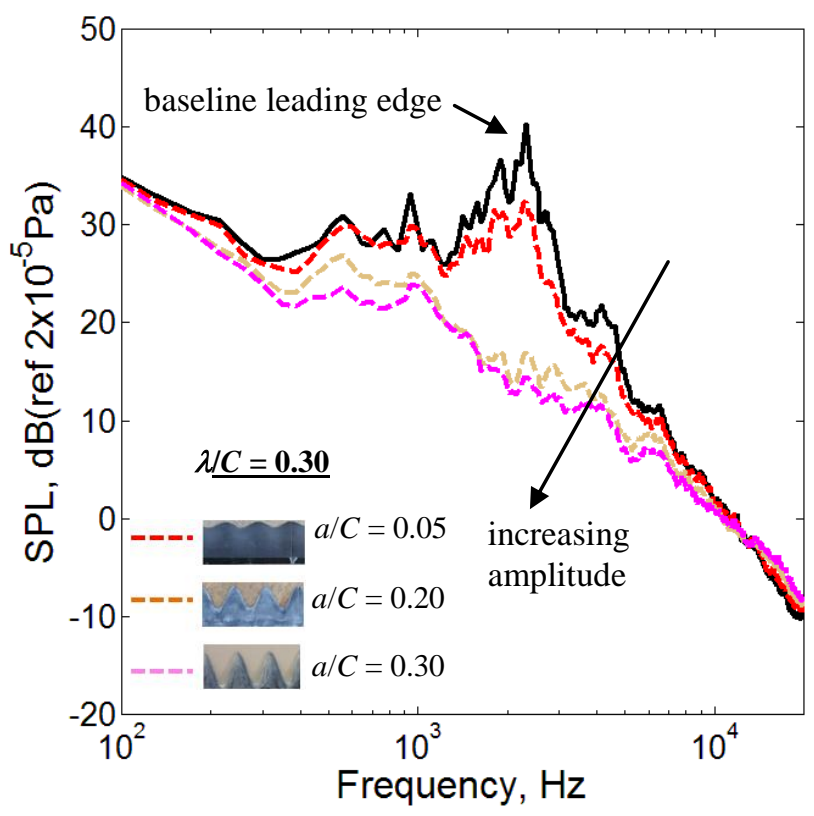

e)
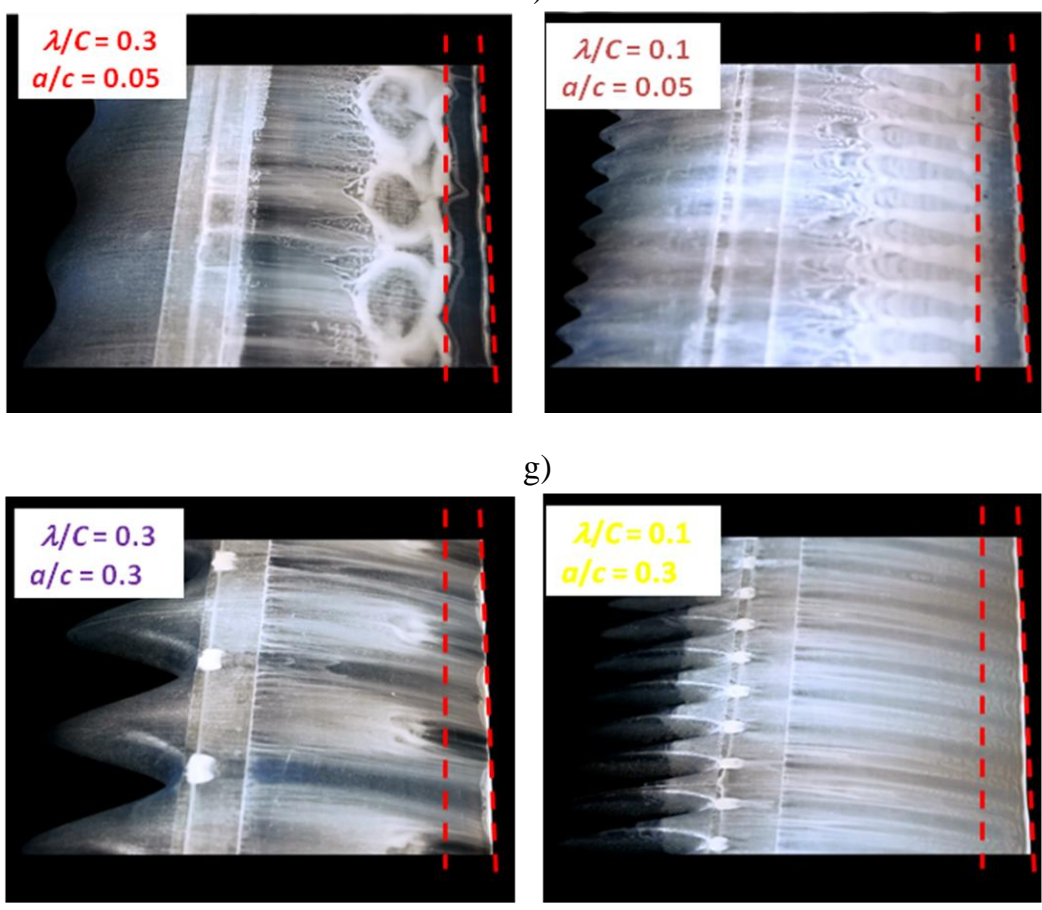

g)

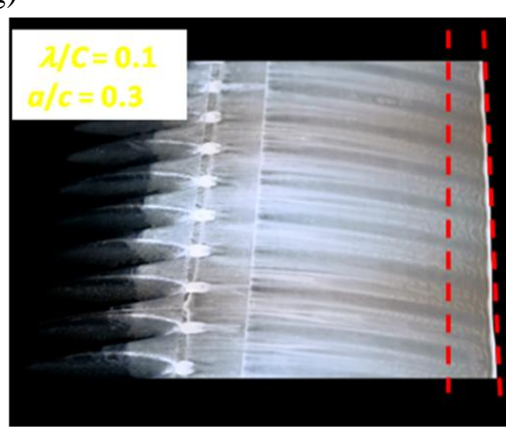

Fig. 5 SPL, dB at $U=32 \mathrm{~ms}^{-1}$, no turbulence grid, for the following sinusoidal leading edges a) variation in wavelength: $\lambda / C=0.30,0.2,0.1$ and $0.05(a / C=0.05)$, and $b)$ variation in amplitude: $a / C=0.05,0.2$ and $0.3(\lambda / C=0.30)$. Surface oil flow visualisation for $c)$ baseline leading edge, and d-g) sinusoidal leading edges of different wavelength and amplitudes. 

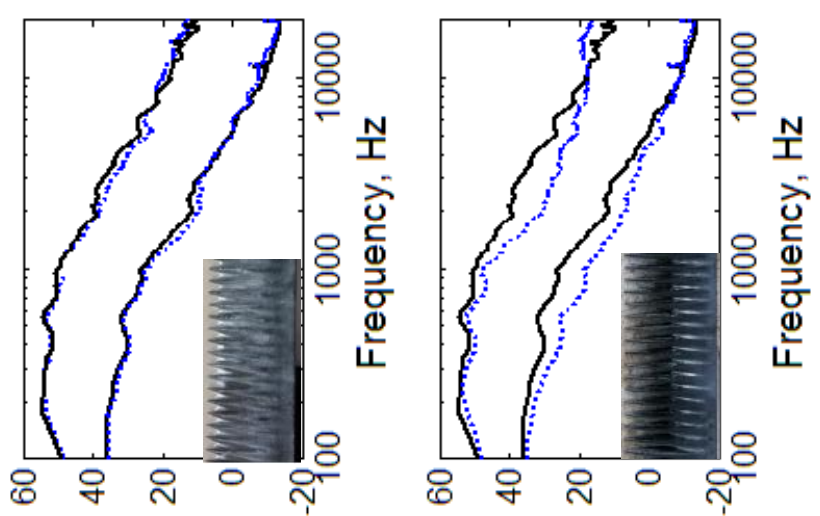

gp 'רds

gp '7ds

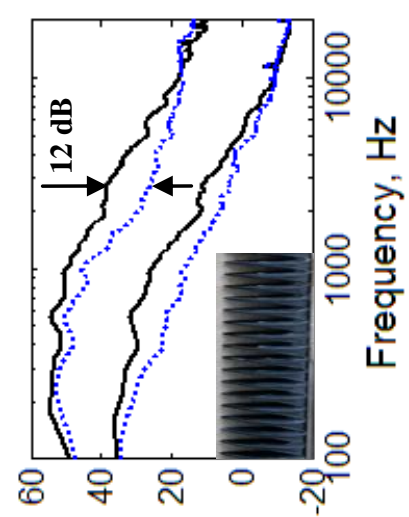

gp 'רds
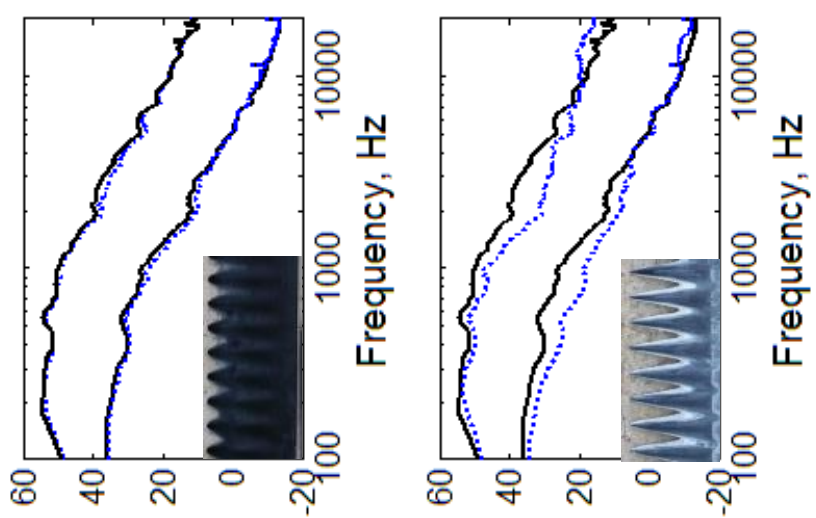

gp 'רds

gp '7ds

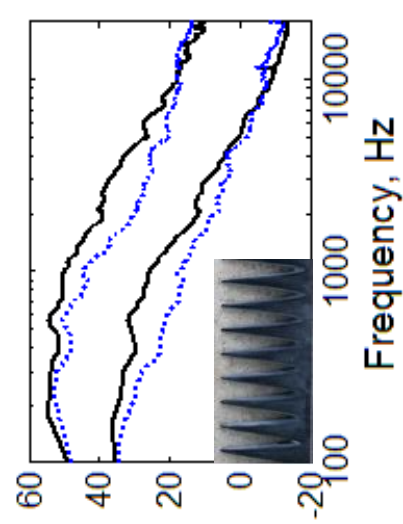

gp 'רds

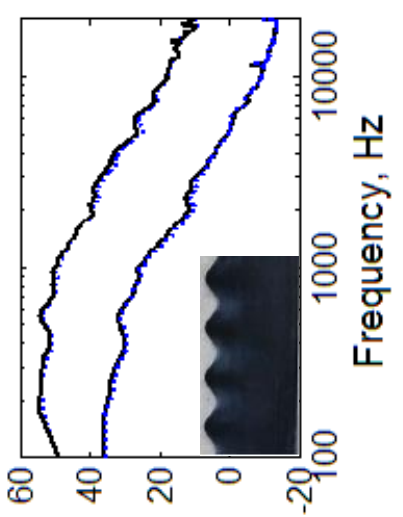

gp 'רdS

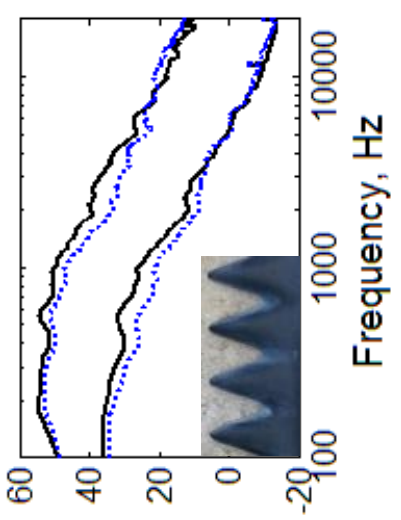

gp 'רdS

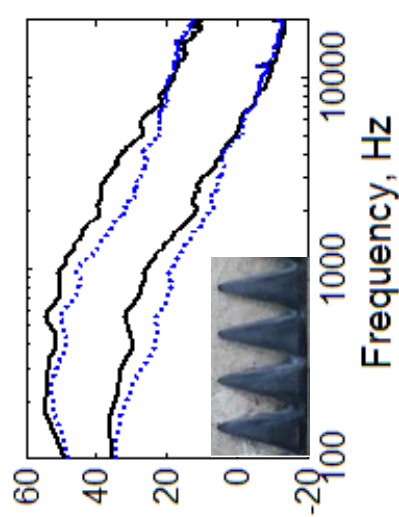

gp '7ds

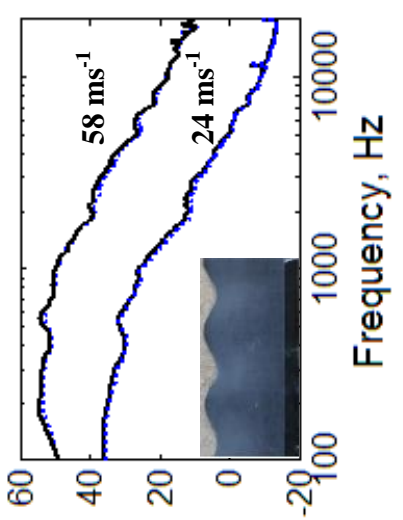

gp 'רds

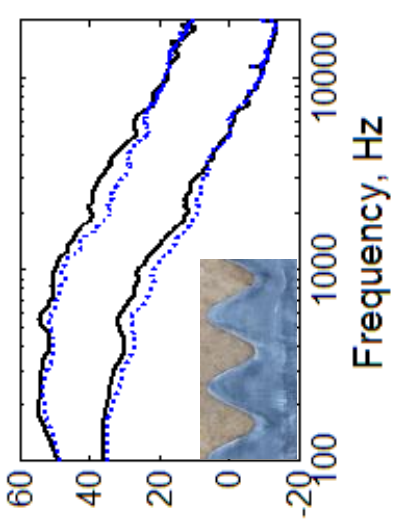

gp 'רds

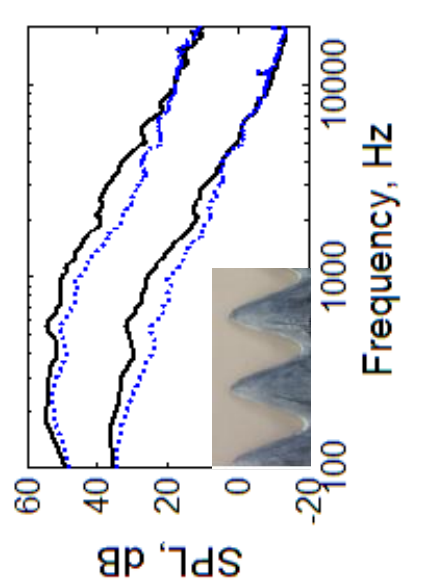

Е

苾

然

刍

焉

웡

I

을

远

辛

'壾

$\infty$

్ㅗㄹ

点

त

II

2

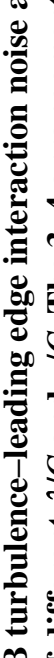

을

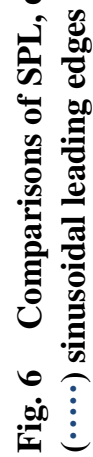

13

American Institute of Aeronautics and Astronautics 

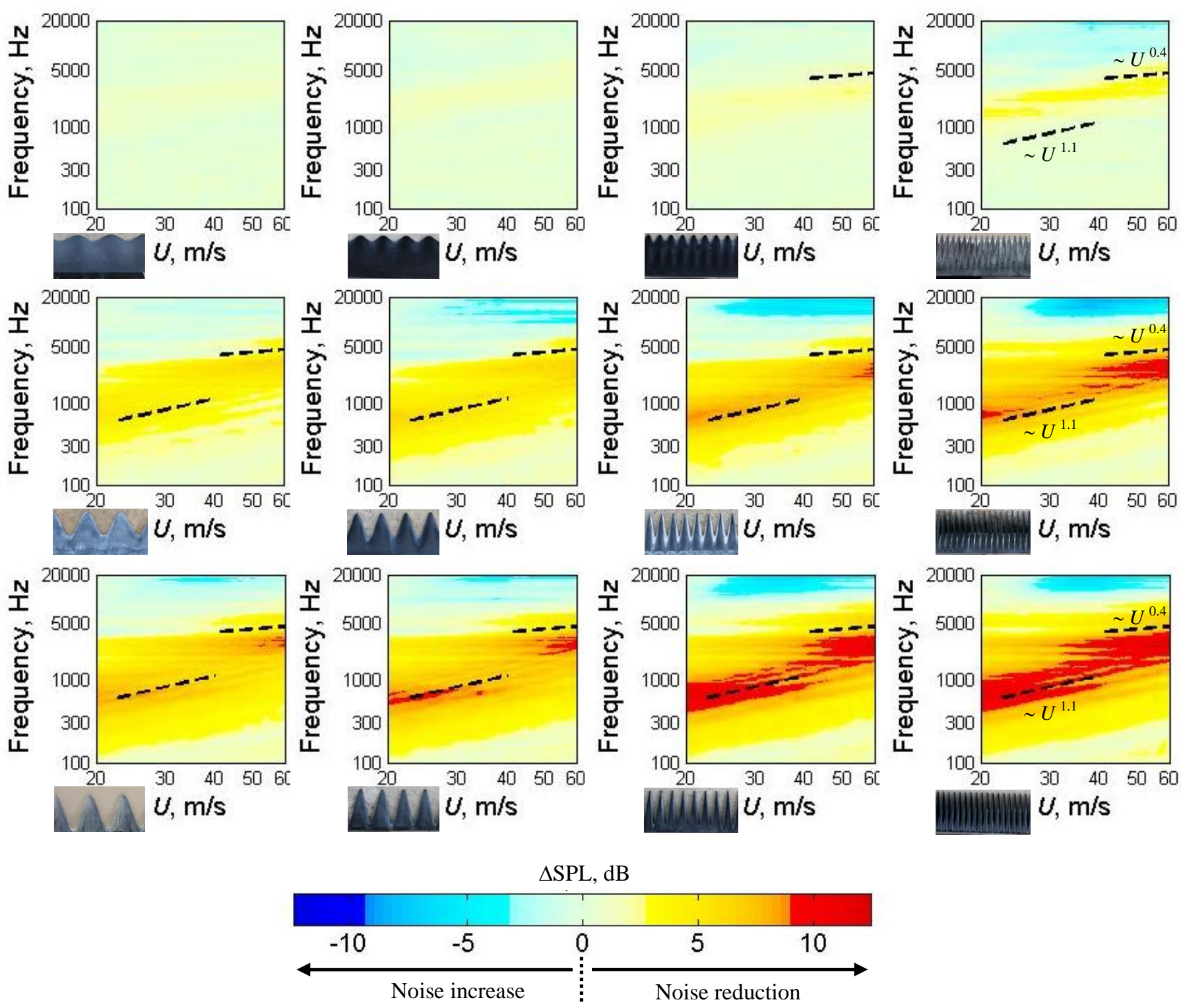

Fig. 7 Contour maps of $\triangle \mathrm{SPL}, \mathrm{dB}$ at different velocities and frequencies, turbulence-leading edge interaction noise, for different sinusoidal leading edges. The $3 \times 4$ arrangement in the figure has the same combination of $\lambda / C$ and $a / C$ as in Fig. 2. 
a)

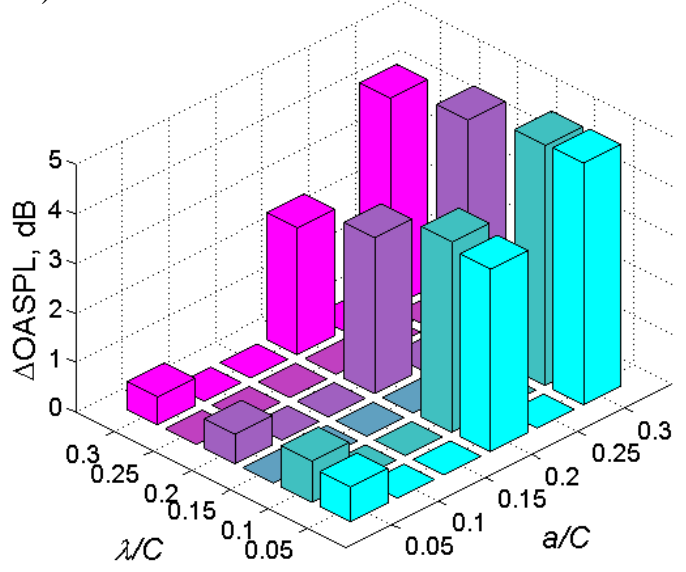

b)

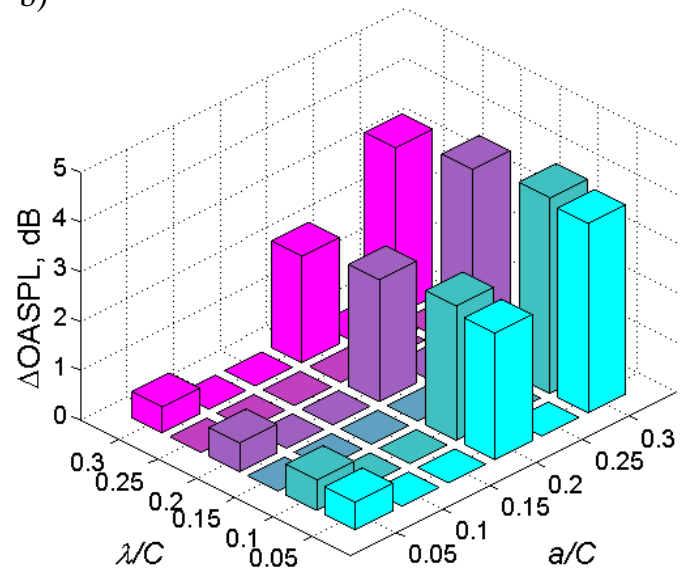

Fig. 8 Bar chart of $\triangle$ OASPL, $\mathrm{dB}$ (noise reduction in the turbulence-leading edge interaction) produced by the sinusoidal leading edges at a) $U=24 \mathrm{~ms}^{-1}$ and b) $U=58 \mathrm{~ms}^{-1}$. 


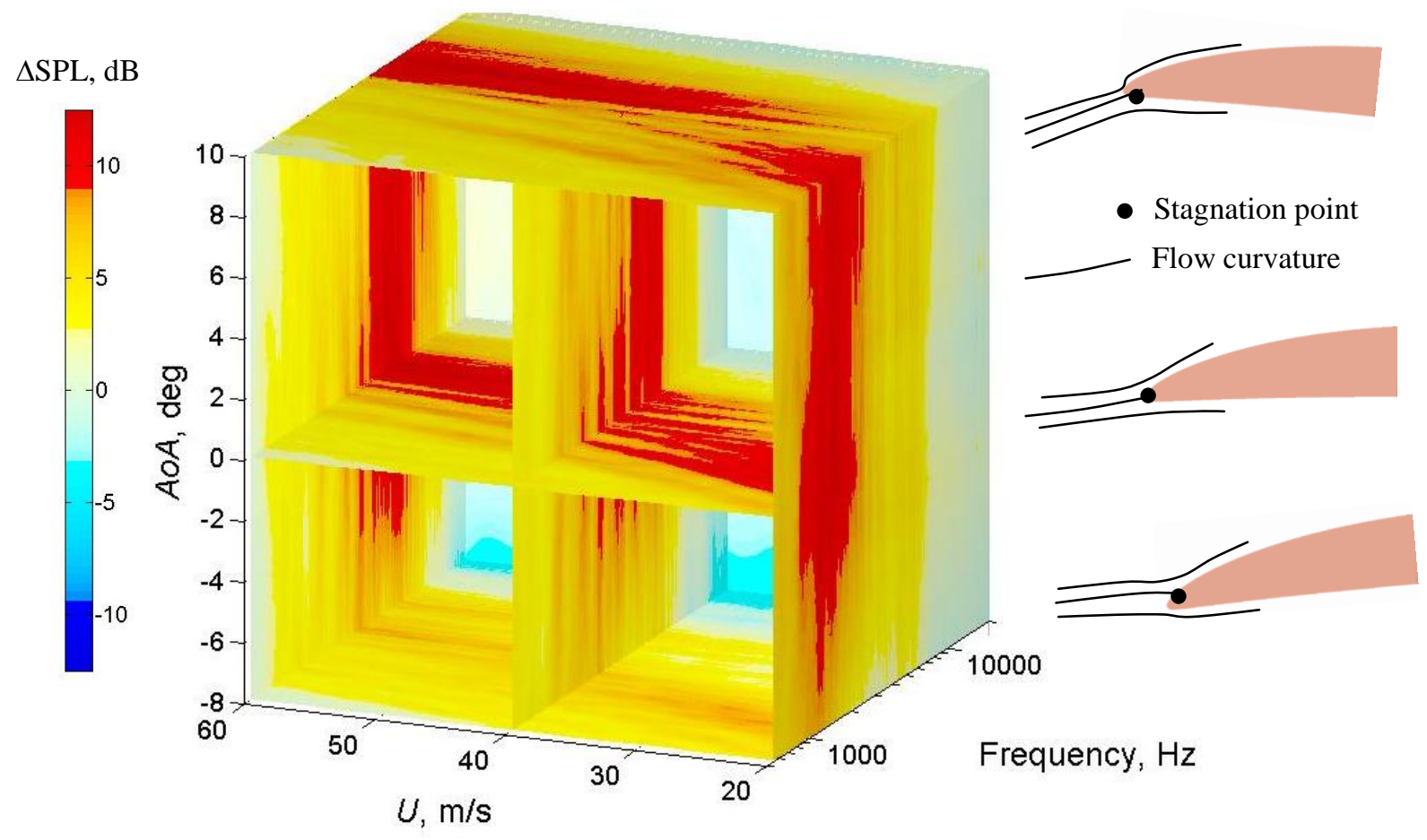

Fig. 9 Contour slices of the $\triangle \mathrm{SPL}, \mathrm{dB}$ at different velocities, frequencies and angles of attack. The sinusoidal leading edge in this case is the $(\lambda / C=0.05 \& a / C=0.3)$ type. 
a)

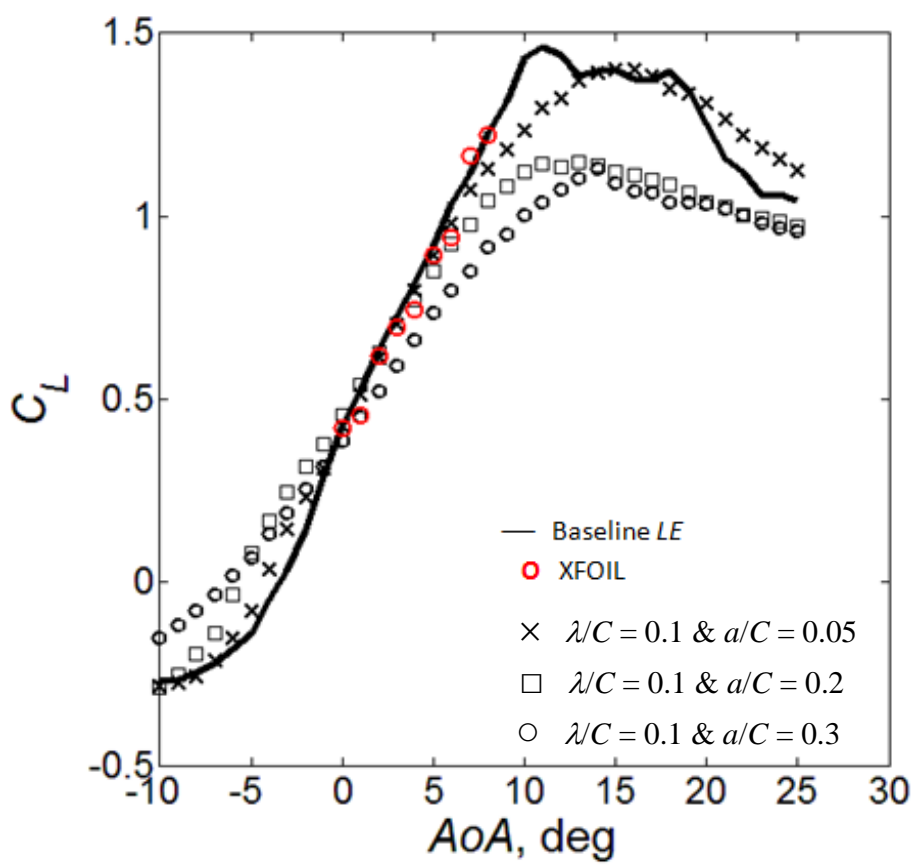

b)

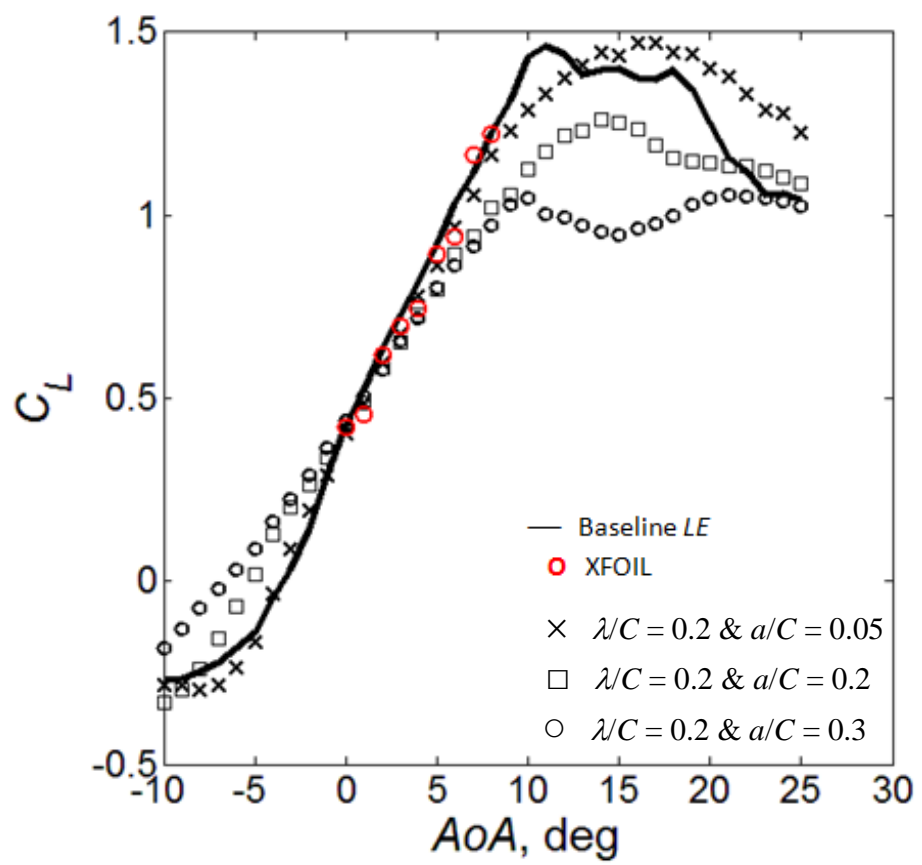

Fig. 10 Lift coefficients $C_{L}$ against angles of attack, deg for the sinusoidal leading edges 
a)

$\lfloor$ Flow direction

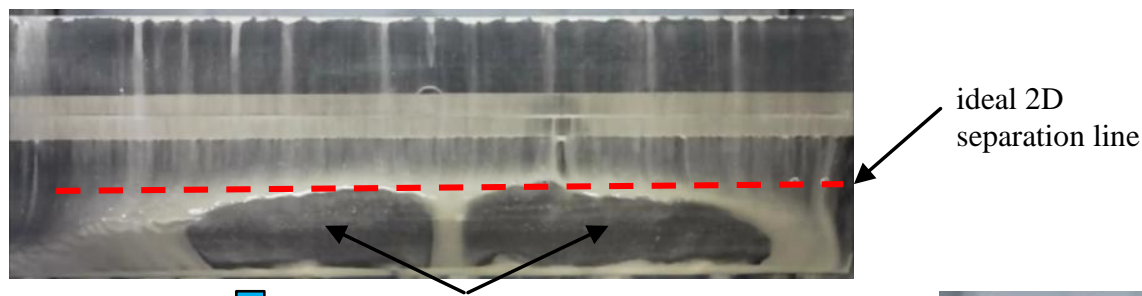

b)

Flow direction $] \quad$ reverse flow regions

c)
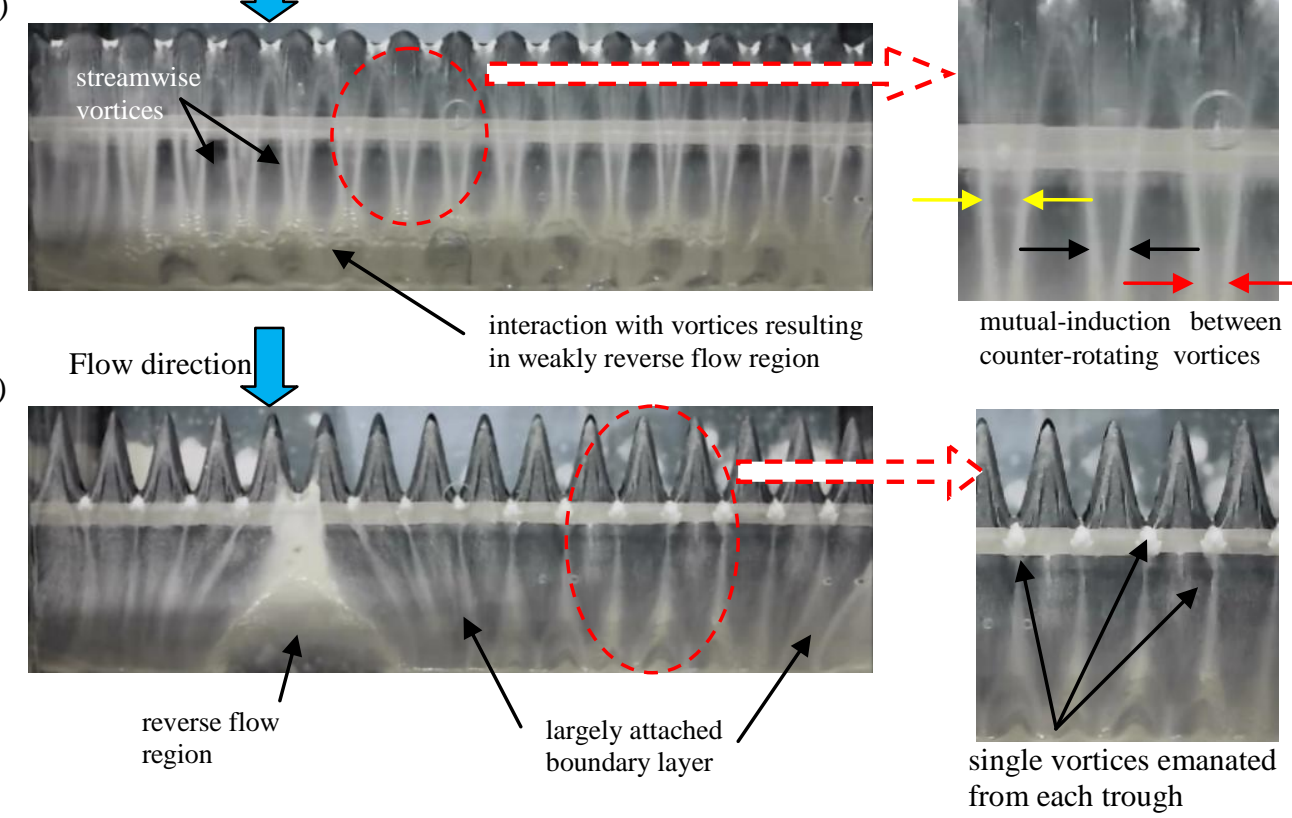

Fig. 11 Surface flow visualisation on the suction side of aerofoil subjected to a) baseline leading edge, b) sinusoidal leading edge of $\lambda / C=0.20, a / C=0.05$ and c) sinusoidal leading edge of $\lambda / C=0.20, a / C=0.30$. The aerofoil is at 10 degree angle of attack at an incoming freestream velocity of $30 \mathrm{~ms}^{-1}$. Experiment was conducted in the aerodynamic wind tunnel. 


\begin{tabular}{|c|c|}
\hline$\lambda / C=0.10, a / C=0.05$ & $\lambda / C=0.20, a / C=0.05$ \\
$\left(\mathbf{0 . 0 9 2 8} \mathbf{~ d e g}^{-\mathbf{1}}\right)$ & $\left(\mathbf{0 . 0 9 6 3} \mathbf{~ d e g}^{-\mathbf{1}}\right)$ \\
\hline$\lambda / C=0.10, a / C=0.20$ & $\lambda / C=0.20, a / C=0.20$ \\
$\left(\mathbf{0 . 0 7 5 3} \mathbf{~ d e g}^{-\mathbf{1}}\right)$ & $\left(\mathbf{0 . 0 7 8 8} \mathbf{~ d e g}^{-1}\right)$ \\
\hline$\lambda / C=0.10, a / C=0.30$ & $\lambda / C=0.20, a / C=0.30$ \\
$\left(\mathbf{0 . 0 6 2 2} \mathbf{~ d e g}^{-\mathbf{1}}\right)$ & $\left(\mathbf{0 . 0 7 0 9} \mathbf{~ d e g}^{-\mathbf{1}}\right)$ \\
\hline
\end{tabular}

Table 1 Summary of the pre-stall lift curve slope for some of the sinusoidal leading edges investigated in this study. Note that the lift curve slope for the baseline straight leading edge is $0.1042 \mathrm{deg}^{-1}$. 